\title{
M-Learning for Teaching in Molding and Casting with Plaster by Using QR Codes
}

\author{
Samart Jabjone and Saisunee Jabjone
}

\begin{abstract}
The objective of this paper is to present the m-learning for teaching in molding and casting with plaster by using QR Codes (MLM) which offers Thai sign language video correspondence to every contents in learning environment. It was developed as a response to stakeholder at Nakhon Ratchasima Rajabhat University, Thailand. The participations in this study were seven deaf and hard hearing student who faced the problem when study in the classroom. This MLM provided the content and video to demonstrate and showed the technique which student could follow and practice by themselves. This MLM used a Universal Design for learning (UDL) approach to explore the iterative development of mobile to meet stakeholder needs. Koole's FRAME model was used to guide the design process, and evaluated their pedagogical effectiveness. The student can access to the lesson by scanning Quick Response $(\mathrm{QR})$ codes. All relative resource can storage using cloud computing. Participants had a positive response to the m-learning. The students and instructors reported enjoying learning with their mobile devices, and found the MLM easy to access and use.
\end{abstract}

Index Terms - Deaf and hard hearing, mobile learning, QR codes, universal design for learning (UDL).

\section{INTRODUCTION}

In Thailand, the deaf and hard hearing students or student with disabilities (for example, study materials that are not in electronic formats, uncaptioned video, PDF files that do not contain any real text and therefore cannot be searched or read aloud by text-to-speech software) are often the same obstacles encountered by students who possess different learning styles. The m-learning with QR codes could benefits many students. Nearly $70 \%$ of students in Nakhon Ratchasima Rajabhat University have their own smartphone. The current capabilities and applications in smartphone consist of making and receiving calls, sending and receiving text messages, basic office tools e.g. calculator, 1luetooth, camera capable of taking stills and video, e-book readers, games, recording audio, GPS/ location aware, and web browser to connect to the internet [1]. By now, it should be clear that with the wide range of mobile phone functionality, there will be many potential uses for mobile devices in education, including the creation and delivery of content. Not directly related to the teaching itself, there are also potential secondary benefits, such as the possibilities for making the teaching environment (smart buildings) more aware of

Manuscript received September 5, 2013; revised November 13, 2013. This work was supported in part by Nakhon Ratchasima Rajabhat University, Thailand.

The authors are with Nakhon Ratchasima Rajabhat University, Nakhon Ratchasima, 30000, Thailand (e-mail: samart.jab@gmail.com, a1102923@hotmai.com). learners based on their mobile phone acting as a beacon or identifier and then both parties having the ability to respond or act on pre-defined inputs and outputs [2]. Mobile learning, or "m-learning", offers modern ways to support learning process through mobile devices, such as handheld and tablet computers, MP3 players, smartphones and mobile phones [3]. It presents unique attributes compared to conventional e-learning: personal, portable, collaborative, interactive, contextual and situated, it emphasizes "just-in-time-learning" as instruction can be delivered anywhere and at anytime through it [4]. Moreover, it is an aid to formal and informal learning and thus holds enormous potential to transform the delivery of education and training. Mobile learning is emerging as one of the solutions to the challenges faced by education [5].

\section{A. Quick Response (QR) Codes}

QR Code is a form of 2D bar codes. "QR" stands for "Quick Response". It was developed by Denso-Wave, a Japanese automatic data capture equipment company [6], in 1994. It is readable by dedicated QR barcode readers and camera telephones. The code consists of black modules arranged in a square pattern on a white background. The information encoded may be text, URL, or other data. A square that consists of black and white squares that looks like an out of focus pixilated image. A QR code is capable of holding 7,089 numeric characters, 4,296 alphanumeric characters, 2,953 binary bytes, 1,817 Kanji characters or a mixture of them. The data capacity is much higher than other 2D codes such as PDF417, DataMatrix and Maxi Code [7]. It stores information in both vertical and horizontal directions. A QR code can be read from any direction in $360^{\circ}$ through position detection patterns located at the three corners as shown in Fig. 1.

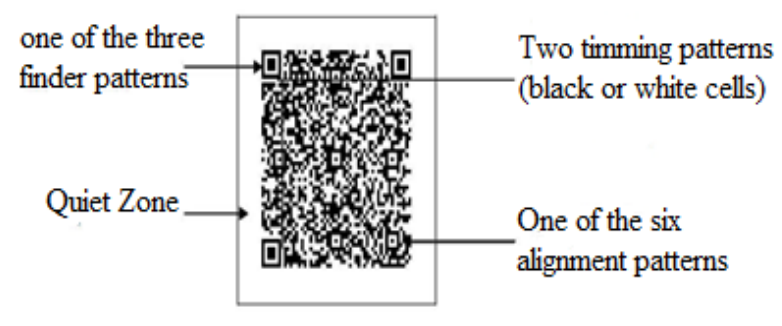

Fig. 1. QR codes.

QR Codes have unlimited potential in the classroom of the $21^{\text {st }}$ century learner [8]. QR Codes can provide an alternative access format for students who need additional support in reading and writing. Using handheld devices e.g. iPad, Smartphone, or a computer students are able to quickly gain access to information while also incorporating the use of their own literacy support apps or software. For example, having trouble typing in a long, complicated URL - use a QR Code 
to navigate to the website instantly without typing. Or, need a read the instructions for a task set by the teacher scan the QR Code created for these instructions using the tablet and use the text-to-speech app. [9].

\section{B. Cloud Learning}

In recent years, cloud computing as a new kind of advanced technology accelerates the innovation for the computer industry. Cloud computing is a computing model based on networks, especially based on the Internet, whose task is to ensure that users can simply use the computing resources on demand and pay money according to their usage by a metering pattern similar to water and electricity consumption. Therefore, it brings a new business model, where the services it provides are becoming computing resources. Cloud computing is highly scalable and creates virtualized resources that can be made available to users. Users do not require any special knowledge about the concept of Cloud computing to connect their computers to the server where applications have been installed and use them. Users can communicate through Internet with remote servers. These servers can exchange their computing slots themselves [10] .Cloud computing is one of the new technology trends likely to have a significant impact on the teaching and learning environment. In Cloud computing, resources can be either externally owned (public Cloud - as provided by Google and Amazon) or internally owned (private Cloud). Public Clouds offer access to external users who are typically billed on a pay-as-you-use basis. The private Cloud is built for the access within the enterprise where the users can utilize the facility without any charge. Mobile Cloud Learning (Fig. 2 ) is a relatively new concept that holds great promises for the future development of education [11]. The two learning modalities can naturally merge, because the characteristics of cloud learning overlaps with mobile learning. The definition of mobile learning has also been evolving, from the early definitions of "learning with mobile devices" to the current one that emphasizes learner mobility resulting from the use of mobile devices. In both definitions, users can acquire learning content from the centralized shared resources and engage in anytime-anywhere context-aware learning via portable devices.

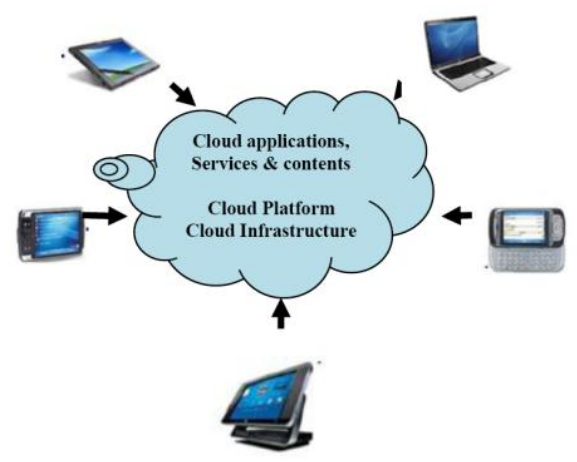

Fig. 2. The concept of mobile cloud learning.

\section{Methodology}

The MLM framework followed in a linear order as show in Fig. 3. It was divided into four phase; analysis, design, development and evaluation. During the Analysis phase, if a mobile learning solution is determined ("YES" line coming out of 1), he or she determines a macrostrategy that includes education or training elements, performance support elements, or combination thereof. He or she also considers the affordances of mobile devices to support the desired learning, in terms of macrostrategy components such as assessment method, motivation enhancement, and opportunities for practice. After determining the m-learning macrostrategy, the designer (during the Design phase) determines the appropriate learning theory given the objectives, learning domain, learning environment, and learning audience (3). The microstrategies are then determined (4). These microstrategies describe the learning activities and how learners will interact with content on the molding and casting with plaster, based on the learning theory chosen. Once the decision is made that an m-learning approach is appropriate (1), the designer injects the appropriate mobile learning considerations (ideally a "ready to go" list that the designer has developed through experience and research) into the steps of MLM (shown by the dotted line "m-learning Considerations" objects). The designer and team leverage these considerations as they encounter them in all phases of the MLM process. If the designer determines that a mobile approach is not called for ("NO" line coming out of 1), he or she continues through the Analysis phase and the rest of the MLM process steps as he or she normally would.

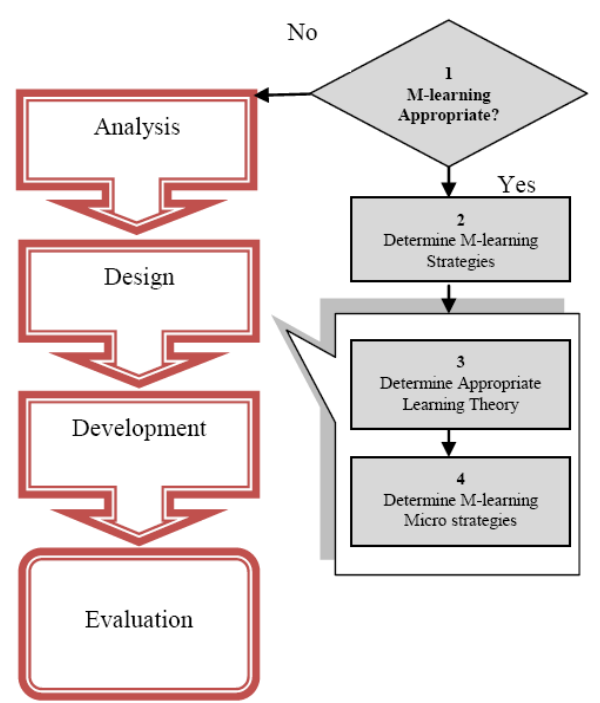

Fig. 3. MLM frame work.

To prepare a document embedded with QR codes, the simplest way to do is to generate the specific codes using some of the tools available from the Internet. These images can then be embedded into the document at the appropriate places. If the mobile device does not build in any QR code reader, the user needs to download the right decoder from the Internet and installs it on to the device. The following steps illustrate the processes.

Step 1: To encode the required text/SMS/URL/Contact into a QR code, we can make use of some Websites that can generate the code for us such as www. qrcode.kaywa.com

Step 2: We can save the generated image file and embed it into wherever we want in the document.

Step 3: We need to load the mobile device with the right decoder which we can find many application websites supplied the decoders for different mobile devices such as 
www.i-nigma.com

Step 4: We need to install the application into the device.

Step 5: For the document with the embedded image, the user can just slide the decoder over the area and the text will automatically be displayed as shown below.

\section{IMPLEMENTATION}

In this research, the MLM was used to replace workbook-based learning for sculpture I course. The universal design was used in MLM. Universal Design for Learning (UDL) is a set of principles that guide the design of inclusive classroom instruction and accessible course materials. UDL's three principles are: 1) multiple methods of representation that give learners a variety of ways to acquire information and build knowledge; 2) multiple means of student action and expression that provide learners alternatives for demonstrating what they have learned; and 3) multiple modes of student engagement that tap into learners' interests, challenge them appropriately, and motivate them to learn [12]. The obstacles faced by deaf and hard hearing students or student with disabilities (for example, study materials that are not in electronic formats, uncaptioned video, PDF files that do not contain any real text and therefore cannot be searched or read aloud by text-to-speech software) are often the same obstacles encountered by students who possess different learning styles, use the latest computer technologies. UDL benefits many students-hence the MLM is in Universal Design for Learning.

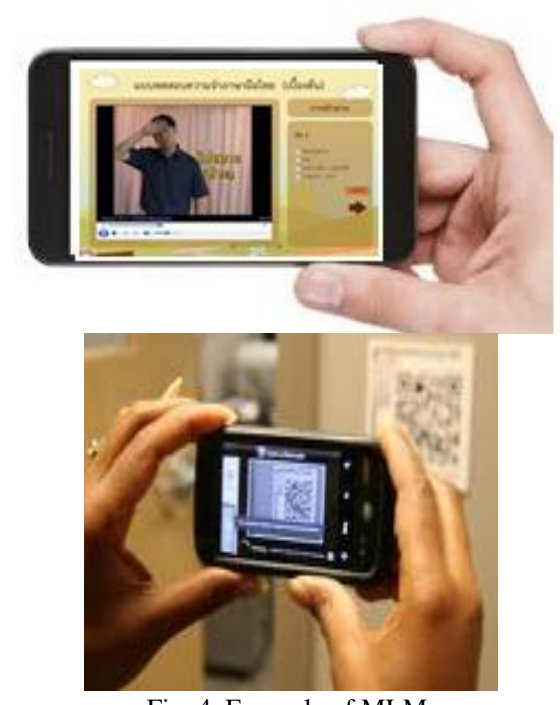

Fig. 4. Example of MLM.

In primer lesson, it was integrated to teach students about QR codes, and provide them with an opportunity to explore the QR code scanning capabilities of their mobile devices. When necessary, instructors and students worked together to locate and install QR code scanning applications. The next two class sessions were used to explore samples of molding and casting with plaster to which QR codes had been mounted. Students were responsible for learning by demonstrate and show the technique via video with sign language. Upon completion of the in-class activities, participating students were invited to complete on online questionnaire about their learning experiences using the QR codes and their own mobile devices. The questionnaires consisted of a combination of fixed and open-response items covering such themes as ease of access, the look and feel of the MLM, levels of interaction with their peers and instructor, and overall impressions. A similar questionnaire was prepared for participating instructors to provide feedback on the learning activities and MLM designs. Responses to fixed and open-response questionnaire items were coded to reflect the research issues. These were analyzed for the identification of major themes related to student and instructor perceptions, and evidence of effects upon transactional distance and the types of activities that form the domains of the FRAME model.

\section{RESULT}

A total of seven students who were deaf or $\mathrm{H} / \mathrm{H}$ and two instructors completed questionnaires during the first phase. All of the students owned smartphones. Four students reported owning two devices, and one student reported owning three (or more). Only two students had a QR code scanning app installed on their devices prior to the study. The remaining respondents were able to download a free app without any reported difficulty. Four students had previously scanned QR codes to access websites, while only one reported previously accessing text-based content, and one reported previously using a QR code to automatically dial a phone number. All of the students indicated that scanning the QR codes was either easy or very easy and that the MLM loaded quickly on their devices. Only one student reported that an MLM did not load properly. All of the students responded that it was easy to view the text and images, and the MLM were easy to navigate. Six students indicated that it was either easy or moderately easy to understand the content, and to complete the "Test Your Knowledge" feedback questions at the end of the lesson. One student indicated that the MLM contained too much information, and that the "Test Your Knowledge" activities were difficult to complete. With respect to interaction with technology, content, peers and instructors, six out of seven student respondents indicated that they shared their mobile devices with another classmate while participating in the MLM activities. Five students and both teachers indicated that they discussed the mobile MLM during the class activities, and four students indicated that they engaged in discussions of the "Test Your Knowledge" activities. All seven students indicated that they viewed the MLM more than once, and five indicated that they showed the MLM to friends outside of the class.

Students and teachers generally indicated that they found the use of the MLM, and their own mobile devices, appealing. Five of the seven students responded that they found these types of learning activities appealing, while one reported a neutral opinion, and one indicated that they found it somewhat unappealing. Only two of the seven students reported having ever used a mobile device for formal learning before, but all of the respondents indicated that they would like to do so again either at school or while on the job. When asked what they liked about using QR code scanners and their own mobile devices to access MLM, students commented on the speed and ease of accessing the learning materials. As one student commented, "it's very easy to scan and find the lesson that you want." 


\section{DISCUSSION}

The results of the first iteration of the MLM show trends in mobile device ownership and the desire to use mobile devices in formal and informal learning. Students and teachers reported enjoying learning with their mobile devices, and found the MLM easy to access and use. Students indicated that they would like to use their mobile devices for learning more often. These results provide a degree of justification to pursue further investigations into integrating m-learning strategies at Nakhon Ratchasima Rajabhat University. But a stronger justification can be provided by grounding these findings in learning theories that explain how the m-learning approach creates an effective learning experience. The Koole's FRAME model [13] provides useful and complimentary lenses for examining the effectiveness of the MLM. Student and teacher responses show a reduction in transactional distance between learners and the content. The content is easy to access and re-access, and it is situated so that it is easier for learners to contextualize the topics. Learner-learner and learner-teacher transactional distance also appear to have been reduced. Data indicate that students interacted with each other and their instructors while participating in the learning activities. The results also indicate that the MLM generated appropriate activity across the domains of the FRAME model. Student and teacher survey responses indicate a high degree of device usability. They also show that learners are actively engaged in social interaction during the learning activities, and that the use of their mobile devices facilitated that interaction by creating a shared situated learning experience, and by generating both formal and informal social discussion. Beyond creating an enjoyable and easily accessible learning experience, the FRAME model illustrates how the uses of the mobile MLM positively affect the learning that is taking place.

There are limitations which must be considered when interpreting the findings of this research. The online questionnaire was the only method of soliciting feedback used in the first phase. The survey schedule would benefit from an in-depth piloting and refinement phase. The addition of either one-on-one or focus group interviews would provide further opportunity to solicit qualitative feedback, and to triangulate the findings with respect to learner perceptions. Data on student achievement on two standardized assessment instruments was collected during the first phase, for comparison with a control class of learners who did not use the MLM. While all learners demonstrated mastery of the required competencies, the sample size was too small to obtain confidence in the results of statistical analyses of the achievement data.

\section{CONCLUSION AND FUTURE WORK}

The integration of information and communication technologies (ICT) in education play an important role to the learning. Information Technology can improve human senses, or substitute one for another. Today computer can do even more, and operate different devices such as home appliances. Even for persons with the most severe challenges, the computer is a helpful tool with which to communicate and control the environment [14]. Mobile technologies offer significant opportunities both for learners and educators in the process of knowledge society. Mobile technologies have the structure of independency of time and place, so mobile technologies have found themselves an important use in e-learning supported by mobile technologies (m-learning) information can be accessible, free from time and place, via mobile technologies which have an opportunity such as wireless communication. By means of their technical features, mobile technologies can be used not only for educational purposes accessing but also for increasing social interaction. Based on their portability and wireless communication features, handheld computers, admitting of verbal and written communication, allow for deaf and hard of hearing students reach communication and learning experience. These technologies are also known assistive technologies for learners in special education context. In this study, we investigated the role that mobile learning play in the learning style of seven deaf and examine how these devices have been effected to their communication and learning patterns. The result showed that m-learning can help deaf and hard of hearing students to use mobile information and communication technologies effectively and independently, and provide them with learning and communication opportunities enriched through mobile technologies. This mobile learning not only follows educational trends but also brings many more benefits, such as: the facilitation of education, a possibility of direct communication between teachers and students, catering for unique students requirements in a personalized way and an opportunity to follow real-time conferences and webinars to interact with professionals right from their homes or classrooms. It also helps to overcome the problems of distance and expenditure and provides instant access to information.

One of the main problems regarding mobile was that until recently the institution had to provide the hardware. However now we are at the stage that we can use the learner's own devices. Additionally, mobile devices can often mean that our teaching and learning materials can be re-purposed and/or usefulness extended which can only be a good thing. It is hoped that future iterations will lead to increased adoption of mobile learning by college instructors. It is also hoped that future iterations will lead to an increased understanding of how m-learning can improve student engagement and learning by reducing transactional distance and increasing activity between students, content, their peers, and technology itself. Looking to the future, the authors would like to expand the scope of the curriculum to include more student participation in a wider range of interactive exercises, thus creating a truly mobile classroom. We are especially encouraged by the possibilities afforded by the plethora of high-powered tablet computing devices that will soon enter the market, many with front facing cameras for video chat and Flash viewing capabilities. A 3G enabled, highly portable tablet device could create a 'classroom' in each student's pocket, and every moment of the day could become a learning event. Additionally encouraging is the upcoming introduction of higher speed $4 \mathrm{G}$ mobile Internet technologies [15]. Faster wireless connections on ultra-portable, always-on devices will allow for a more connected Rural Scholars experience. Such connectivity would allow more learners to engage in deeper learning experiences, no matter how remote their location. Ultimately, this could equate to better care of our in-need rural populations. 


\section{ACKNOWLEDGMENT}

This research was made possible by supporting from the president of Nakhon Ratchasima Rajabhat University and Research and Development Institute.

\section{REFERENCES}

[1] M. Ford and T. Leinonen, MobilED - mobile tools and services platform for formal and informal learning, Edmonton, AB: Athabasca University Press, 2009, pp. 198.

[2] J. Gregson and D. Jordaan, Exploring the challenges and opportunities of m-learning within an international distance education program, Edmonton, AB: Athabasca University Press, 2009, pp. 215.

[3] D. S. Metcalf, mLearning: Mobile learning and performance in the palm of your hand, Amherst, MA: HRD Press, 2006, pp. 125.

[4] C. Bradley, R. Haynes, J. Cook, T. Boyle, and C. Smith, Design and evelopment of multimedia learning objects for mobile phones, Edmonton, AB: Athabasca University Press, 2009, pp. 157-159.

[5] A. Ramsden, The use of QR codes in education: A getting started guide for academics, Bath, U.K.: University of Bath, 2008, pp. 189-195.

[6] N. Pachler, B. Bachmair, and J. Cook, Mobile Learning: structures, Agency, Practices, New York: Springer Press, 2010, pp. 216.

[7] U. Kremer, J. Hicks, and J. Rehg, "A Compilation Framework for Power and Energy Management on Mobile Computers," in Proc. 2001 INTERMAG Conf., August 2001, pp. 115-119.

[8] E. Lawrence, L. Naismith, P. Lonsdale, G. Vavoula, and M. Sharples, Literature Review in Mobile Technologies and Learning, U.K.: University of Birmingham, 2005, pp. 256-285.

[9] M. G. Moore, The theory of transactional distance, NJ: Mahwah, 2007, pp. 89-105.

[10] A. Holzinger, A. Nischelwitzer, and M. Meisenberger, "Mobile Phones as a Challenge for m-Learning: Examples of Mobile Interactive Learning Objects (MILOs)," in Proc. 3th Annu. IEEE International
Conference on Pervasive Computing and Communications, Kauai Island (HI), 2005, pp. 309.

[11] J. Yao and Y.-X. Sun, "The Initial Idea of New Learning Society which Based on Cloud Computing," Modern Educational Technology, vol. 20, no. 1, pp.14-17, May 2010 .

[12] B. Connell, M. Jones, R. Mace, J. Mueller, A. Mullick, E. Ostroff, J. Sanford, E. Steinfeld, M. Story, and G. Vanderheiden, The principles of universal design, Raleigh, NC: Center for Universal Design, North Carolina State University. 1997, pp. 245-258.

[13] M. L. Koole, A model for framing mobile learning, Edmonton, AB: AU Press, 2009, pp. 38.

[14] E. Cuervo, A. Balasubramanian, D. Cho, A. Wolman, S. Saroiu, R. Chandra, and P. Bahl, "Making Smartphones Last Longer with Code offload," in Proc. 8th INTERMAX Conf., June 2010, pp. 49-62.

[15] A. Ramos and J.Triñona, Distance education technologies in Asia; New Delhi: Sage, 2010, pp. 132-152.

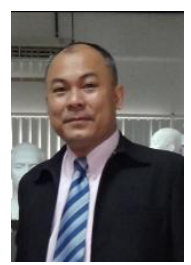

Samart Jabjone was born in May, 1971. He graduated doctoral degree from Ubon Ratchatani, Thailand, major in applied art and design. He is an assistant professor at the Fine Art program, Nakhon Ratchasima Rajabhat University, Thailand. His research interest is in the area of Fine Art, Applied Art, Art and Cultural, Sociology, Political and education technology.

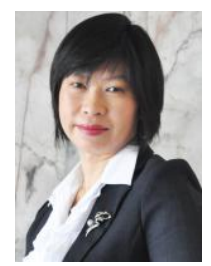

Saisunee Jabjone born in March, 1972. She graduated doctoral degree from the University of Technology, Sydney, Australia, major in computing. She is an assistant professor at the Informatics program, Nakhon Ratchasima Rajabhat University, Thailand. Her research interest is in the area of data mining, data warehouse, ontology, data management, database, bioinformatics, and education technology. 